\title{
Fine structure and oscillations of a quiescent prominence
}

\author{
N. Petrov ${ }^{1}$, P. Duchlev ${ }^{1}$, B Rompolt ${ }^{2}$, and P. Rudawy ${ }^{2}$ \\ ${ }^{1}$ Institute of Astronomy, Bulgarian Academy of Sciences, Bulgaria; \\ ${ }^{2}$ Astronomical Institute of the Wroclaw University, Poland
}

\begin{abstract}
Series of $\mathrm{H}_{\alpha}$ spectra and slit-jaw $\mathrm{H}_{\alpha}$ filtergrams of a quiescent prominence (QP) taken at Pic du Midi Observatory on 7 November 1977 are studied. The image processing of the $\mathrm{H}_{\alpha}$ filtergrams reveals an internal structure of the prominence consisting of several arches. Series of high-resolution $\mathrm{H}_{\alpha}$ spectra obtained with the slit located on selected parts of one of the prominence arches chosen for Doppler shift analysis. The prominence arch show cyclic displacement along the line-of-sight (L.O.S.) direction implying Alfven string-mode oscillations.
\end{abstract}

\section{Observations and method of data processing}

We examine the structure and the L.O.S. velocity variations of a $\mathrm{QP}$ on the base of high-resolution $\mathrm{H}_{\alpha}$ spectra and filtergrams obtained by one of us (BR) at Pic du Midi Observatory on 7 November 1977. The prominence was located at the NE (N45-E) limb, according to Meudon synoptic map for rotation N1681. The filament had lasted for two solar rotations and during the observations it had been on its first rotation. The series of high-resolution $\mathrm{H}_{\alpha}$ spectra have been recorded on photographic film using the 11-m solar horizontal telescope equipped with a 9-m spectrograph and a spectrohelioscan developed at the Astronomical Institute of the Wroclaw University. Slit-jaw units coupled with an $\mathrm{H}_{\alpha}$ camera have provided $\mathrm{H}_{\alpha}$ filtergrams together with the actual slit position against the prominence body. The spectra from the QP have been taken with an exposure time of $5 \mathrm{~s}$ and $0.75 \mathrm{~mm}$ slit width. The $\mathrm{H}_{\alpha}$ filtergrams have revealed that the QP consists of several arches. For the purpose of this study we took seventeen $\mathrm{H}_{\alpha}$ spectra obtained at a chosen slit position located on one of the arches, marked by a line 12 in Figure 1 . All the spectra have been obtained in a time interval of about $57 \mathrm{~min}$ (from 10:07:48 to 11:04:35 UT) with a nearly equal time step of $3.5 \mathrm{~min}$ between the consecutive scans.

\section{Results and discussions}

As it can be traced in the Figure 1 (upper part), the sign and the magnitude of the L.O.S. velocities are changing along the slit, and with the time, especially near the periphery of the arch. The maximum magnitude of the velocity is about $(1.5 \mathrm{~km} / \mathrm{s}$. In order to trace cyclic displacements or irregular oscillations of the prominence arch along the L.O.S., which could be accepted as parallel to the solar surface, we compute, for the selected prominence volumes position of the centre of gravity of each $\mathrm{H}_{\alpha}$ profile with respect to the corresponding chromospheric $\mathrm{H}_{\alpha}$ profile. As a representative statistical quantity for the determined displacement we take the average of the velocity distribution along the slit. We calculated these values for two separated volumes at the slit position 12. For the upper part of the prominence (upper arch) we calculated the L.O.S. velocities as one separated volume and the lower part (lower arch) as another volume. The behavior 

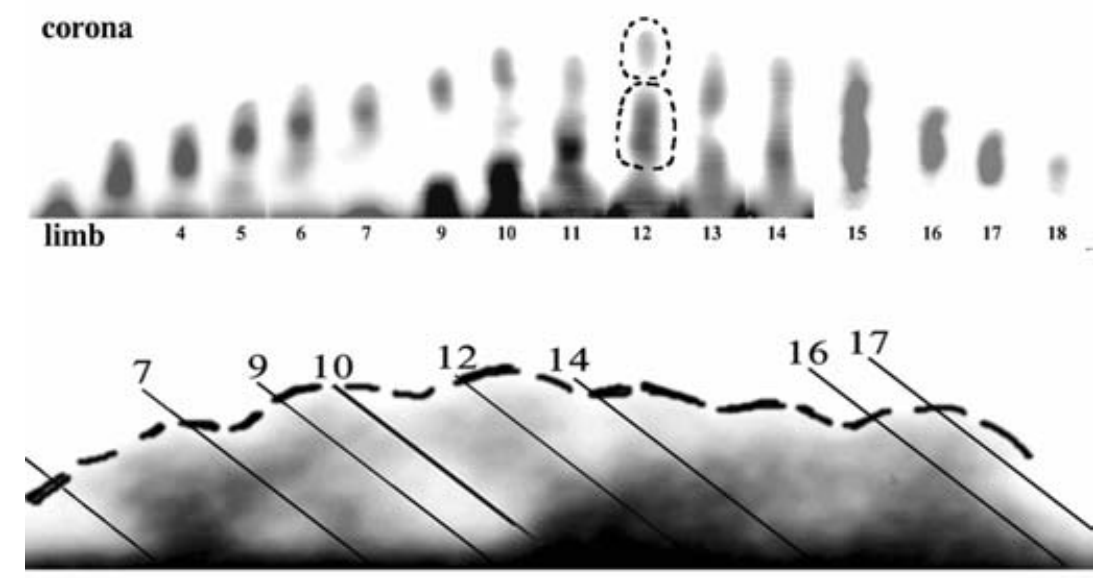

Figure 1. One series of $\mathrm{H}_{\alpha}$ spectra used for Doppler shift measurements (upper part) on the entire prominence body. $\mathrm{H}_{\alpha}$ filtergram of the prominence (lower part). The position along the slit is given in $50 \mu$ (one pixel) step equal to $343 \mathrm{~km}$ on the Sun.

of the averaged L.O.S. velocities of the upper part is the same as these upper volumes but at different slit positions. The tendency implies oscillation of the prominence arch with a frequency $\omega=310^{-4} \mathrm{~s}^{-1}$. The results were presented by Petrov, Dermendjiev, and Rompolt, (1998) and Detchev et al. (1999) and Dermendjiev et al. (2001).

To keep for a long time the structure of a prominence body we need a state of a stable equilibrium. In this case in order to have a QP the following equations should be fulfiled: $p=$ const $-1 / 2 \rho V_{a}^{2}$ and $\delta V_{a}^{2} / \delta t=0$, where $p$ is plasma pressure, $\rho$ - density, and $V_{a}$ is Alfven speed. The Alfven speed $V_{a}$ is proportional to the magnetic field $B$ : $V_{a} \approx B$. The period of plasma oscillations in a magnetic tube is: $T=L / V_{a}$, where $L$ is the length of the magnetic tube. Let we use one typical values of $B=8 \mathrm{Gs}$ for QPs and $V_{a}=55 \mathrm{~km} / \mathrm{s}$, then for the two periods of oscillations that we calculated we have: $L_{\text {upper }} \approx 200000 \mathrm{~km}, L_{\text {lower }} \approx 130000 \mathrm{~km}$. These are two lengths of two different arches fine structure of the QP. The Doppler shift measured with respect to the averaged $\mathrm{H}_{\alpha}$ profile of the chromosphere indicates cyclic displacement or vibration of the arches that could be referred to Alfven string modes. Using the average of the consecutive in time L.O.S. velocity along the slit as a representative estimate for tracing cyclic displacement, we find frequencies $\omega=310^{-4} \mathrm{~s}^{-1}$ and $\omega=210^{-4} \mathrm{~s}^{-1}$ respectively.

\section{Acknowledgements}

This work is partially supported by the National Scientific Found (Bulgaria) under Grant 1004/00. B.R. would like to thank to Dr. J.-L. Leroy for the invitation at Pic du Midi Observatory and kind hospitality and to Dr. Z. Mouradian for kind permission to use the Meudon solar horizontal telescope to the Pic du Midi Observatory.

\section{References}

Dermendjiev V. N., Petrov N., Detchev M. Tz., Rompolt B., and Rudawy P. 2001 Solar Phys. 202, p. 99.

Detchev, M. Tz., Dermendjiev, V. N., Petrov, N. I., and Rompolt, B.: 1999, in A. Wilson (ed.), Magnetic Fields and Solar Processes, ESA SP-448, p.485.

Petrov, N. I., Dermendjiev, V. N., and Rompolt, B.: 1998, JOSO Ann. Report 1997, p.145. 